\title{
Observation of monolayer valence band spin-orbit effect and induced quantum well states in $\mathrm{MoX}_{2}$
}

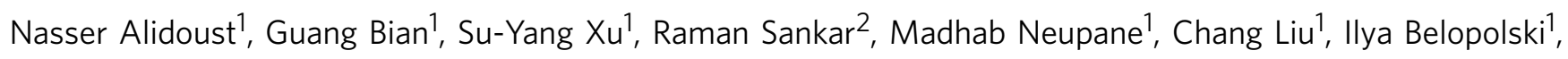
Dong-Xia $\mathrm{Qu}^{3}$, Jonathan D. Denlinger ${ }^{4}$, Fang-Cheng $\mathrm{Chou}^{2} \&$ M. Zahid Hasan ${ }^{1}$

Transition metal dichalcogenides have attracted much attention recently due to their potential applications in spintronics and photonics because of the indirect to direct band gap transition and the emergence of the spin-valley coupling phenomenon upon moving from the bulk to monolayer limit. Here, we report high-resolution angle-resolved photoemission spectroscopy on $\mathrm{MoSe}_{2}$ single crystals and monolayer films of $\mathrm{MoS}_{2}$ grown on highly ordered pyrolytic graphite substrate. Our experimental results resolve the Fermi surface trigonal warping of bulk $\mathrm{MoSe}_{2}$, and provide evidence for the critically important spin-orbit split valence bands of monolayer $\mathrm{MoS}_{2}$. Moreover, we systematically image the formation of quantum well states on the surfaces of these materials, and present a theoretical model to account for these experimental observations. Our findings provide important insights into future applications of transition metal dichalcogenides in nanoelectronics, spintronics and photonics devices as they critically depend on the spin-orbit physics of these materials.

\footnotetext{
${ }^{1}$ Joseph Henry Laboratory, Department of Physics, Princeton University, Princeton, New Jersey 08544, USA. ${ }^{2}$ Center for Condensed Matter Sciences, National Taiwan University, Taipei 10617, Taiwan. ${ }^{3}$ Condensed Matter and Materials Division, Lawrence Livermore National Laboratory, Livermore, California 94550, USA. ${ }^{4}$ Advanced Light Source, Lawrence Berkeley National Laboratory, Berkeley, California 94305, USA. Correspondence and requests for materials should be addressed to M.Z.H. (email: mzhasan@princeton.edu).
} 
$\mathrm{n}$ the recent years, with the discovery of graphene ${ }^{1,2}$ and topological insulators ${ }^{3-8}$, there has been an outburst of research activities to understand the physical properties of two-dimensional (2D) materials and their applications to optoelectronics and spintronics. One family of 2D materials that has recently attracted much attention is transition metal dichalcogenides (TMDCs) such as monolayers and few-layers of $\mathrm{MX}_{2}(\mathrm{M}=\mathrm{Mo}, \mathrm{W} \text { and } \mathrm{X}=\mathrm{S}, \mathrm{Se})^{9,10}$. These materials are easy to exfoliate and fabricate ${ }^{11,12}$, and have band gaps comparable in size to that of silicon $(1.1-1.4 \mathrm{eV}$ in bulk and $1.5-1.9 \mathrm{eV}$ in monolayers) with the predicted property of transitioning from an indirect to a direct one upon reduction in size from bulk to monolayer ${ }^{9,13-19}$, making them remarkably suitable for potential use in electronics applications such as thin film transistors ${ }^{20}$. More excitingly, it has been predicted that the exotic spin-valley coupling phenomenon occurs in monolayers of TMDCs, thus making them appropriate for spintronics and valleytronics devices $^{21-24}$. Nevertheless, even though many recent studies have examined the possibility of using TMDCs in single-layer transistors $^{25-28}$, and have investigated their optical properties and potential use in optoelectronics $18,19,23,29-32$, surprisingly there has only been extremely limited experimental work on uncovering the key aspects of the electronic band structures of bulk, fewlayers and monolayers of these materials and their modification under external effects such as doping, surface deposition or heterostructuring. The few existing momentum-resolved spectroscopic studies on these materials also lack the resolution and depth to highlight the important features of their band structures ${ }^{33-37}$. For instance, only recently, using micro-angleresolved photoemission spectroscopy (ARPES), the evolution of the valence band at the corner of the Brillouin zone (BZ) from higher to lower binding energies upon reducing the number of layers to monolayer has been demonstrated ${ }^{37}$.

The interest in monolayers of the $\mathrm{MX}_{2}$ series lies in the emergence of the direct band gap as a consequence of the disappearance of van der Waals interlayer interactions in the monolayer limit ${ }^{9,19}$, as well as the valence band strong spin-orbit splitting because of the loss of inversion symmetry $9,21,22,37$. However, as films of TMDCs on typical substrates such as $\mathrm{Si} / \mathrm{SiO}_{2}$ grow in very small flakes of $\sim 10 \times 10 \mu \mathrm{m}^{2}$ and are usually composed of islands of variable thicknesses next to each other, tracking these two properties in a momentum-resolved manner has proven to be challenging. Furthermore, the splitting of the valence band top into two distinct degenerate bands in the bulk limit due to both strong spin-orbit coupling and interlayer hopping ${ }^{38}$ could be of interest and has not been resolved to date. Moreover, until very recently $\mathrm{MoS}_{2}$ has experimentally gotten the most attention among various members of the TMDCs family, such as $\mathrm{MoSe}_{2}, \mathrm{WS}_{2}$, and $\mathrm{WSe}_{2}$. This is despite the fact that the stronger spin-orbit coupling of the latter compounds make them more attractive for spintronics applications.

Thus, in this work, we utilize ARPES to directly map the electronic band structure of bulk $\mathrm{MoSe}_{2}$ across the entire BZ. We then study the evolution of its band structure upon in situ surface deposition with an alkali metal (potassium). Our results demonstrate the formation of a nearly free $2 \mathrm{D}$ electron gas (2DEG) within the potassium overlayers confined to the surface of $\mathrm{MoSe}_{2}$ in the form of robust quantum well states (QWSs). This quantum confinement of potassium layers could potentially enhance the optoelectronics performance of bulk and thin films of the TMDCs and be utilized in optoelectronics applications of these materials. We also resolve the band structure of a monolayer of $\mathrm{MoS}_{2}$ grown on highly ordered pyrolytic graphite (HOPG) substrate using the chemical vapour deposition (CVD) growth method and find evidence suggesting the existence of the split valence band induced by strong spin-orbit coupling and broken inversion symmetry. We compare our ARPES results with our first-principles theoretical calculations to gain microscopic insights on the electron behaviour in these materials, which is critically important for optimizing their potential performance in device settings.

\section{Results}

Crystal structure of $\mathbf{M X}_{\mathbf{2}}$. The crystal structure of $\mathrm{MX}_{2}(\mathrm{M}=\mathrm{Mo}$, $\mathrm{W}$ and $\mathrm{X}=\mathrm{S}, \mathrm{Se}$ ) is a layered structure with layers of $\mathrm{M}$ atoms sandwiched between two layers of $\mathrm{X}$ atoms. These layers, which are held together through van der Waals forces, are shifted relative to each other in such a way that $\mathrm{M}$ atoms in one layer are placed directly above the $\mathrm{X}$ atoms in the neighbouring layers $9,10,34$. This crystal structure and the corresponding BZ are shown in Fig. 1b,c, respectively. The centre of the BZ is the $\Gamma$ point, with $M$ points the midpoints of the hexagonal $B Z$ edges, and $K$ points the corners of the hexagon. $\bar{\Gamma}, \bar{M}$ and $\bar{K}$ are the projection of these points onto the $2 \mathrm{D}$ surface $\mathrm{BZ}$ as illustrated in Fig. 1c. X-ray photoemission spectroscopy measurements of the single crystals of $\mathrm{MoSe}_{2}$ used in our studies reveal sharp peaks corresponding to Mo $3 s, 3 p$ and $3 d$ core levels, as well as those of Se $3 p$ and $3 d$ as shown in Fig. 1a, thus indicating the high quality of the studied samples.

Electronic structure of bulk $\mathrm{MoSe}_{2}$. We start by investigating the band structure of $\mathrm{MoSe}_{2}$. The $k-E$ maps of the electronic band structure measured by ARPES (left panels in Fig. 2a,b) along the two high symmetry directions of the $\mathrm{BZ}, \bar{\Gamma}-\bar{M}$ and $\bar{\Gamma}-\bar{K}$, show extraordinary resemblance to the calculated bands from the firstprinciples calculations (right panels in Fig. 2a,b). The ARPES measurements here were performed at a photon energy of $h v=90 \mathrm{eV}$. The kinetic energy value of the Fermi level is determined by measuring that of the polycrystalline gold separately at the same photon energies and under the same experimental conditions. The valence band maximum (VBM) is found to be located at the $\bar{\Gamma}$ point of the BZ at a binding energy of $1.2 \mathrm{eV}$. The valence band just below the topmost band at binding energies of $\sim 1.7-1.9 \mathrm{eV}$, which appears clearly in our first-principle calculations, has a somewhat weaker intensity in our ARPES measurements because of matrix element effects.

One important feature of our measurements is the unambiguous observation of the two distinct bands near the top of the valence band along the $\bar{\Gamma}-\bar{K}$ direction of the BZ. These bands are highlighted in Fig. 2c, a zoomed-in version of the top part of the valence band along the $\bar{\Gamma}-\bar{K}$ direction, and are well in agreement with band structure calculations conducted here as well as the ones reported in earlier studies ${ }^{9,14,15}$ (see Supplementary Note 1 and Supplementary Figs 1-3 for more details). Constant binding energy contours at representative binding energies of $1.4,1.7$ and $2.0 \mathrm{eV}$ are presented in Fig. $2 \mathrm{~d}-\mathrm{f}$. At $E_{\mathrm{B}}=1.7 \mathrm{eV}$, there exists only one electron-like pocket around each of the $\bar{K}$ points of the BZ (see Fig. 2e), whereas two concentric pockets are observed at $E_{\mathrm{B}}=2.0 \mathrm{eV}$ (see Fig. 2f), clearly resolving the two distinct bands at the $\mathrm{BZ}$ corners. Moreover, the hole pockets near $\bar{K}$ exhibit trigonal warping effect as seen in Fig. 2e,f. This effect has been theoretically predicted in monolayer TMDCs ${ }^{39,40}$, and here we have shown that it exists in the bulk materials as well.

Spin-orbit-induced splitting of the valence band in monolayer TMDCs. Figure 1d shows our first-principles band structure calculations for bulk (top row) and monolayer (bottom row) $\mathrm{MoS}_{2}$, with (right column) and without (left column) considering spin-orbit coupling. These demonstrate the indirect to direct band gap transition on going to the monolayer limit and the 


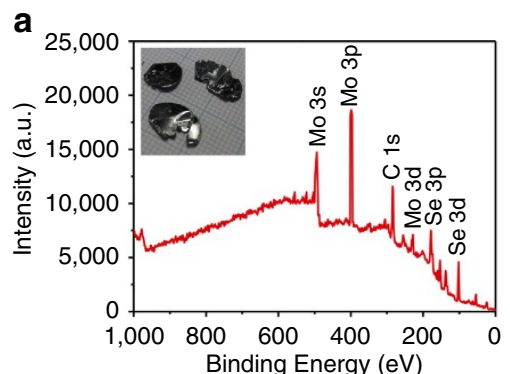

b

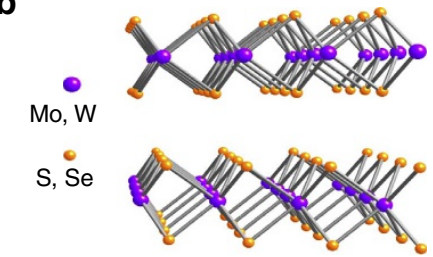

C

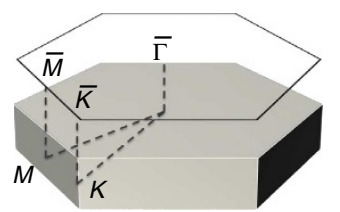

d
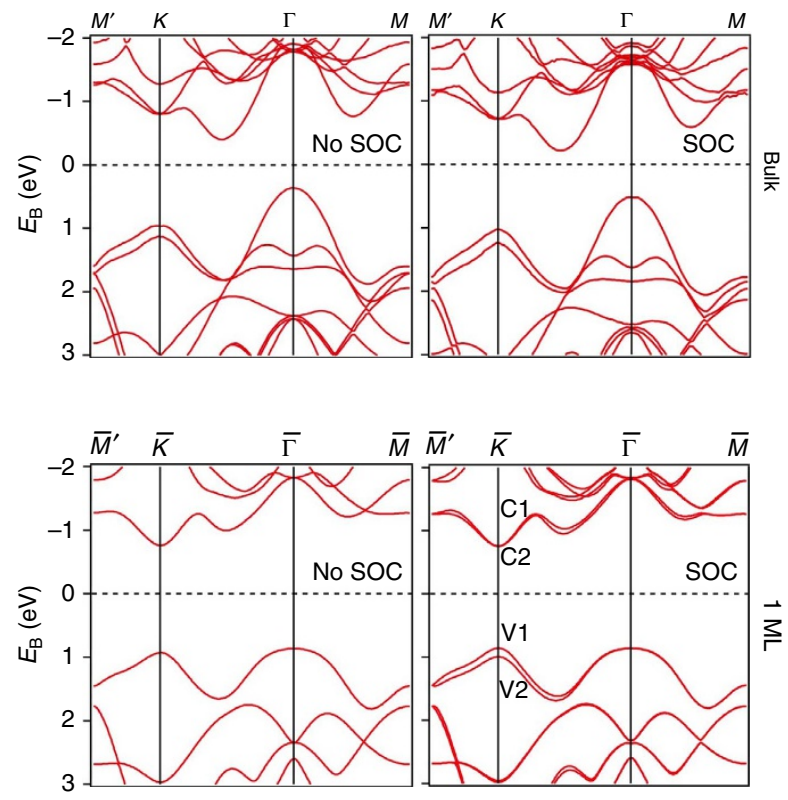

Figure 1 | Electronic structure of transition metal dichalcogenides (TMDCs). (a) X-ray photoemission spectroscopy measurement of the studied $\mathrm{MoSe}_{2}$ crystals, showing both Mo and Se peaks. The inset shows a photograph of these crystals. (b) Three dimensional crystal structure of layered TMDCs, with the chalcogen atoms $(X)$ in orange and the metal atoms $(M)$ in purple. (c) The corresponding bulk and surface Brillouin zone (BZ) with the high symmetry points marked. (d) First-principles band structure calculations for bulk (top row) and monolayer (bottom row) MoS 2 with (right column) and without (left column) considering spin-orbit coupling, demonstrating the indirect to direct band gap transition on going to the monolayer limit and the spin-orbit splitting of the monolayer valence band. The Fermi level is indicated by the dashed lines.

spin-orbit splitting of the monolayer valence band in TMDCs. The Fermi level in these $k-E$ maps is indicated by the dashed lines. These results are in good agreement with the previously reported first-principles calculations on this material ${ }^{21,41}$.

In the next step, we turn our attention to monolayer $\mathrm{MoS}_{2}$ on HOPG substrate. This conducting substrate was deliberately chosen to avoid the electronic charging of the film during the photoemission process. The $k-E$ map of the electronic band structure of this sample along the high symmetry direction $\bar{\Gamma}-\bar{K}$ presented in Fig. 3a, reveals the electronic bands of monolayer $\mathrm{MoS}_{2}$ as well as the linear $\pi$ bands of graphite from the HOPG substrate at the $\bar{K}$ point of the BZ. We have conducted firstprinciples calculations of the hybrid structure of monolayer $\mathrm{MoS}_{2}$ and graphite (Fig. 3b), which indicates the general agreement with our ARPES findings. Despite the existence of the graphite's $\pi$ bands at the $\bar{K}$ points, and their merging with the $\mathrm{MoS}_{2}$ valence band around these points of the $\mathrm{BZ}$, we can still resolve a large section of the $\mathrm{MoS}_{2}$ valence band top near $\bar{K}$ along the high symmetry direction $\bar{\Gamma}-\bar{K}$, as highlighted in Fig. 3c, a zoomed-in version of Fig. 3a near the top of the valence band.

Moreover, we note the broadening of the valence band top along the $\bar{\Gamma}-\bar{K}$ high symmetry direction (see Fig. 3c). Performing $2 \mathrm{D}$ curvature analysis ${ }^{42}$ of the obtained bands, we resolve two split branches of the valence band of $\mathrm{MoS}_{2}$ along this high symmetry direction (close to the $\bar{K}$ point) as shown in Fig. $3 \mathrm{~d}$. The green dotted lines are guides to the eyes for these two spinorbit split branches of the valence band. This is consistent with the spin splitting scenario predicted to exist in this material, as shown by our first-principles calculations in Fig. 3e. Monolayers of TMDCs lack inversion symmetry and are noncentrosymmetric. The in-plane confinement of electron motion and high mass of the elements in these materials result in strong spin-orbit splitting of the valence band. This property makes these materials promising candidates for applications in spintronics devices $9,21,22,24$. Our observation here is the first momentum-resolved experimental evidence for such spin-orbit splitting. Further, spin-resolved ARPES studies are needed to identify the spin configuration of these split bands.

To further demonstrate the orbital property and spin texture of monolayer $\mathrm{MoS}_{2}$, we calculate the charge densities and spin expectation values of the lowest two conduction states and the topmost two valence states at $\bar{K}$ marked in Fig. 1d as C1, C2, V1 and V2, respectively. The charge density plots shown in Fig. $3 \mathrm{f}$ indicate that the dominant orbital components are Mo $4 d_{3 z^{2}-r^{2}}$ and Mo $4 d_{x^{2}-y^{2}}+4 d_{x y}$ for $\mathrm{C} 1(\mathrm{C} 2)$ and $\mathrm{V} 1(\mathrm{~V} 2)$, respectively. The calculated spin expectation values of states near $\bar{K}$ show nearly out-of-plane spin polarization, which can be attributed to an interplay of the pure in-plane electron motion and the inversion symmetry breaking crystal potential. The out-of-plane character of Mo $4 d_{3 z^{2}-r^{2}}$ explains the negligible spin splitting between the $\mathrm{C} 1$ and $\mathrm{C} 2$ states.

On the other hand, the calculated energy splitting between V1 and V2 states is $138 \mathrm{meV}$ at $\bar{K}$, as a consequence of the prominent in-plane charge density variation of Mo $4 d_{x^{2}}-y^{2}+4 d_{x y}$ orbital, which is in good agreement with previous results from density functional theory (DFT) calculations ${ }^{21,41}$. According to the calculated spin expectation values, we deploy a straightforward method to determine the spin orientation of the V1 and V2 states, which are important when considering the selection rule of polarized photon excitations. Viewing monolayer $\mathrm{MoS}_{2}$ from top, as sulphur atoms sit on the hexagonal lattices it is alway possible to find a primitive vector connecting to adjacent sulphur atoms in the same direction as $\bar{\Gamma}-\bar{K}$. Calling this vector a and drawing another vector $\mathbf{b}$ from the sulphur atom at the origin of $\mathbf{a}$ to the nearest Mo atom, the spin orientation of V2 is in the direction of $\mathbf{a} \times \mathbf{b}$ and that of V1 points to the opposite direction (Fig. 3g). 

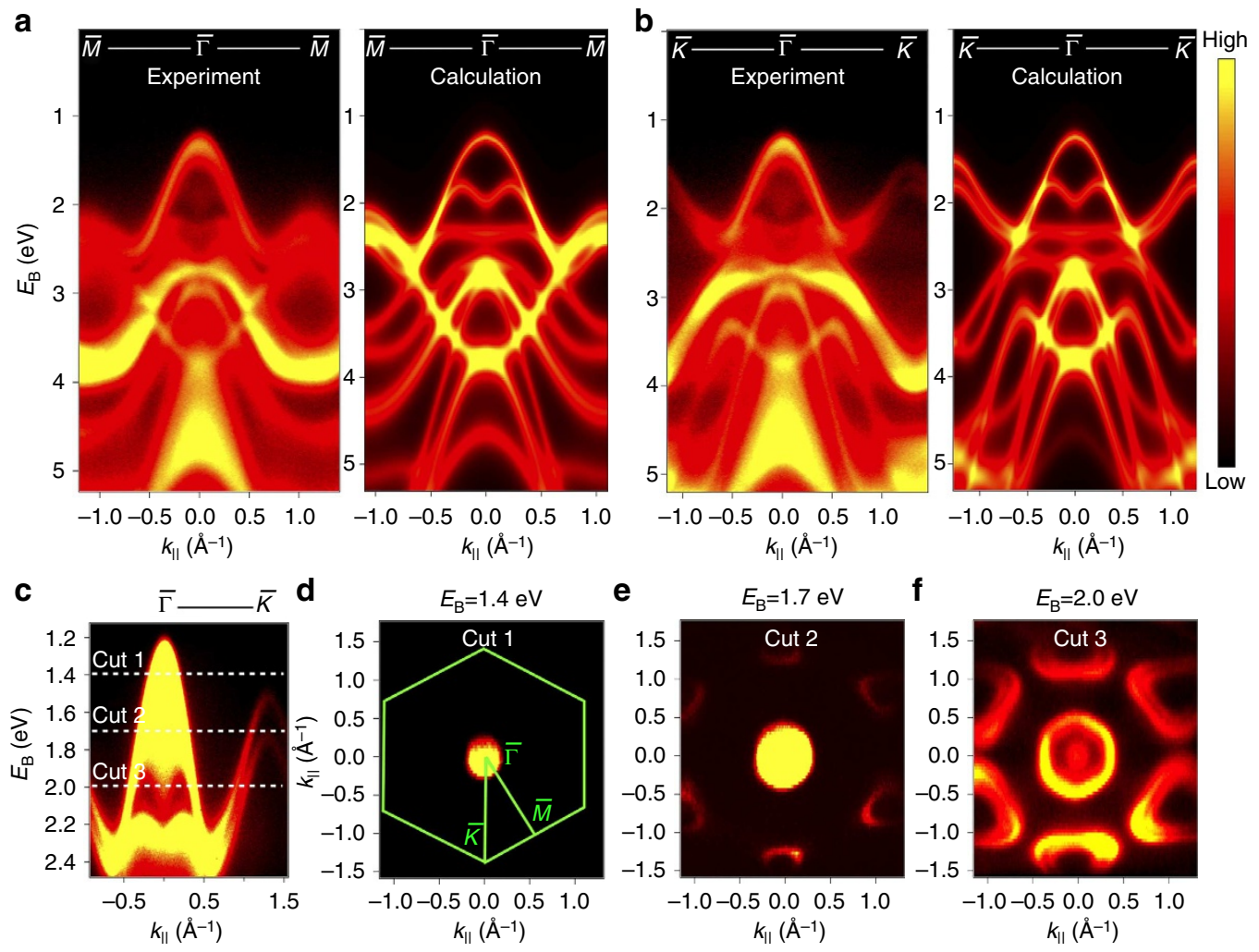

Figure 2 | Electronic structure and spin-degenerate bands of bulk $\mathbf{M o S e}_{\mathbf{2}}$. (a) Angle-resolved photoemission spectroscopy (ARPES) electronic structure measurements (left panel) and first-principles calculated electronic structure (right panel) of $\mathrm{MoSe}_{2}$ crystals along the high symmetry direction $\bar{\Gamma}-\bar{M}$. (b) Same as a for measurements and calculations along the high symmetry direction $\bar{\Gamma}-\bar{K}$. (c) Zoomed-in version of the ARPES electronic structure spectra along the $\bar{\Gamma}-\bar{K}$ direction near the top of the valence band, highlighting the two distinct spin-degenerate bands at the $\bar{K}$ point of the $B Z$. (d-f) Constant binding energy contours at $E_{B}=1.4,1.7$ and $2.0 \mathrm{eV}$, which are indicated in panel c. The green hexagon in $\mathbf{d}$ represents the first $\mathrm{BZ}$. The two distinct bands can also be distinguished in the contour at $E_{\mathrm{B}}=2.0 \mathrm{eV}$ in $\mathbf{f}$ from the electron-like pockets around the $\bar{K}$ points. The ARPES measurements were performed at photon energy of $h v=90 \mathrm{eV}$ and temperature of $T=20 \mathrm{~K}$.

Observation of QWSs of a nearly free 2DEG by surface deposition. To study the effect of alkali metal deposition on the surface of bulk $\mathrm{MoSe}_{2}$, we deposit potassium (K) in situ on cleaved surfaces of crystals of this material, and track the evolution of the band structure spectra as the deposition occurs. Deposition was performed at $T=60 \mathrm{~K}$ with an estimated deposition rate of $\sim 0.08-0.09 \AA \mathrm{min}^{-1}$. The total deposition time is $1 \mathrm{~h}$ and the nominal thickness of potassium is about 4.8-5.4 $\AA$. Figure $4 \mathrm{a}$ shows the evolution of the band structure $k$ $E$ map along the $\bar{\Gamma}-\bar{M}$ direction of the BZ during the deposition process. We observe that the VBM at $\bar{\Gamma}$ moves closer to the Fermi level by about $200 \mathrm{meV}$. We associate this shift with the formation of a conducting layer on the surface of our sample after potassium deposition. More specifically, the VBM of the $\mathrm{MoSe}_{2}$ sample is at $E_{\mathrm{B}}=1.0 \mathrm{eV}$, but before deposition is shifted down by about $200 \mathrm{meV}$ because of the electronic charging effect (a photoninduced effect, not intrinsic to the material under study, but rather caused by the photoemission process ${ }^{43}$ ). Next, we observe the formation of new electronic states in the band gap of $\mathrm{MoSe}_{2}$, extending from the Fermi level to the valence band (see middle panels in Fig. 4a). Upon the completion of the deposition process these in-gap states are completely formed as shown in the last panel of Fig. 4a and b.

We note the formation of electron-like pockets on the Fermi surface after the completion of the deposition process. These pockets appear right at the $\bar{K}$ points of the $\mathrm{BZ}$. As the energy spacing between these pockets and the VBM $(0.8 \mathrm{eV})$ is smaller than the indirect gap of $\mathrm{MoSe}_{2}(\sim 1.1 \mathrm{eV})$, we rule out the possibility of these pockets being a part of the conduction band of $\mathrm{MoSe}_{2}$. The resulting Fermi surface after potassium deposition shows two concentric circular-shaped pockets around the centre of the BZ, as well as the aforementioned pockets at the $\bar{K}$ points (Fig. $4 \mathrm{~d}$ ). The circular pockets around $\bar{\Gamma}$ are identified as the first and second QWSs, whereas the pockets at $\bar{K}$ are possibly resulting from the surface states of potassium. We furthermore conduct first-principles calculations of potassium layers formed on the surface of bulk $\mathrm{MoSe}_{2}$, as shown in Fig. 4c. In the simulation, the relaxed interlayer spacing of potassium is determined to be $3.51 \AA$, and so the nominal thickness corresponds to $1.35-1.53$ layers of freestanding $\mathrm{K}$ films. As an independent check of this value, we compare the ARPES spectra with DFT simulations, which gives a thickness between 1 and 2 layers, in agreement with the previous estimate (for more details see Supplementary Note 1 and Supplementary Fig. 4). The possibility of directly accessing the conduction band of bulk and monolayer TMDCs through intercalation and adsorption of alkali metals has been theoretically predicted ${ }^{44}$. Our results suggest that adsorption of alkali metals on the surface of bulk $\mathrm{MoSe}_{2}$ results in the formation of QWSs and a nearly free 2DEG rather than electron doping and providing access to the conduction band.

\section{Discussion}

The splitting of the valence band along the $\bar{\Gamma}-\bar{K}$ high symmetry direction of the monolayer $\mathrm{MoS}_{2}$ and other TMDCs, which is due to strong spin-orbit coupling (because of the high mass of the 
a

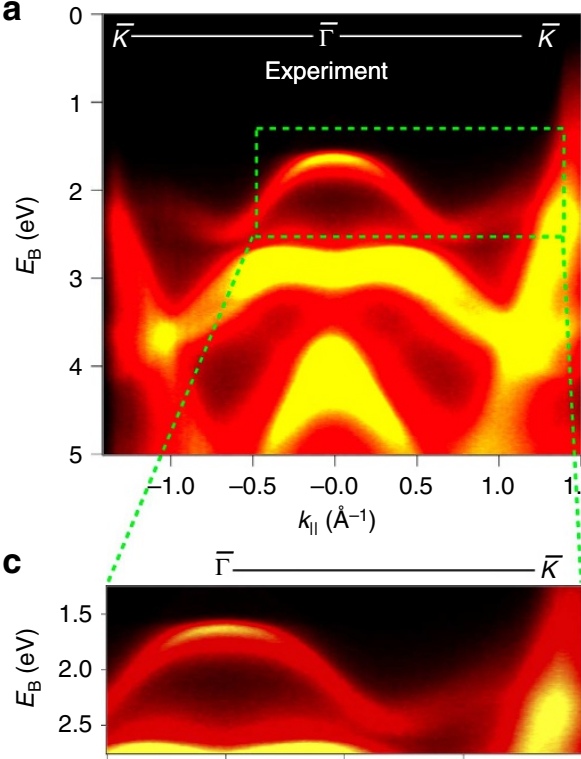

d

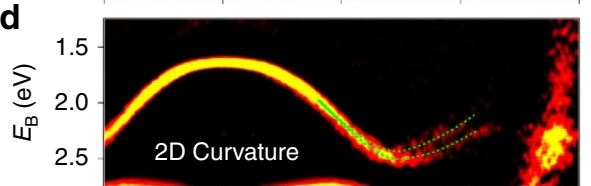

e

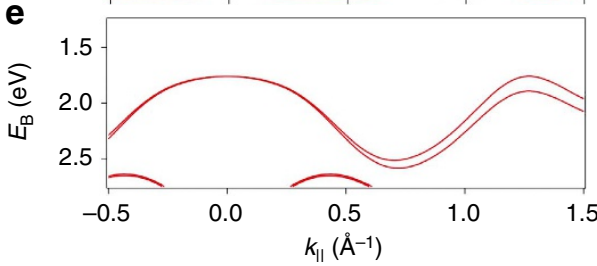

b
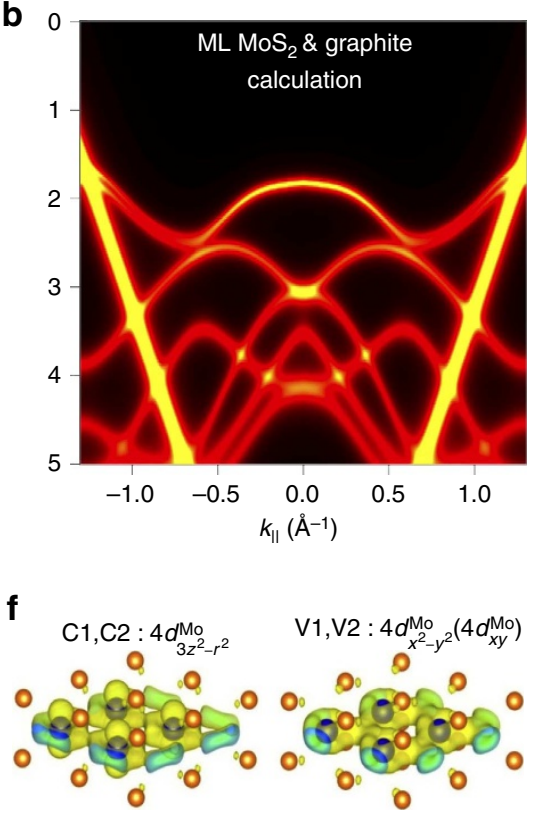

9

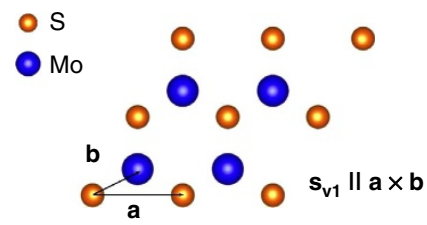

Figure 3 | Spin-orbit-induced splitting of the valence band in monolayer $\mathbf{M o S}_{\mathbf{2}}$. (a) ARPES electronic structure measurements of monolayer MoS $\mathbf{S}_{2}$ on highly ordered pyrolytic graphite (HOPG) substrate along the high symmetry direction $\bar{\Gamma}-\bar{K}$, showing the observation of $\mathrm{MoS}_{2}$ monolayer electronic bands as well as the $\pi$ bands of graphite at the $\bar{K}$ point of the BZ. (b) First-principles calculated electronic structure measurements of monolayer MoS 2 accompanied by graphite along the high symmetry direction $\bar{\Gamma}-\bar{K}$. (c) Zoomed-in version of the ARPES electronic structure spectra along the $\bar{\Gamma}-\bar{K}$ direction. (d) 2D curvature plot of the bands near the valence band top, showing the experimental observation of the predicted spin-orbit split valence band along $\bar{\Gamma}-\bar{K}$. The green dotted lines are guides to the eyes for these two spin-orbit split branches of the valence band. Here the ARPES measurements were performed at photon energy of $h v=110 \mathrm{eV}$ and temperature of $T=200 \mathrm{~K}$. (e) First-principles calculated bands near the valence band top, confirming the experimental observation of the predicted spin-orbit split valence band. (f) Charge densities of conduction and valence states at $\bar{K}$. (g) Top view of monolayer $\mathrm{MoS}_{2}$. The spin polarization of the $\mathrm{V} 1$ band is in the direction of $\mathbf{a} \times \mathbf{b}$, provided that $\mathbf{a}$ is aligned along the $\bar{\Gamma}-\bar{K}$ direction.

elements) and lack of inversion symmetry, has been suggested in previous theoretical studies as well as our first-principles calculations here ${ }^{9,21}$. For the bulk, this splitting is caused by the combination of interlayer interaction and spin-orbit coupling. In this case, there is no spin splitting between these two bands and they are indeed spin-degenerate (Kramers' degeneracy) because of the presence of inversion symmetry along with time reversal symmetry. But, the strong spin-orbit coupling in TMDCs results in enhanced splitting of these spin-degenerate bands at the $K$ point of the BZ (see Fig. 1d) ${ }^{38}$. Our results resolve these two distinct bands at the corners of the BZ through ARPES measurements and theoretical first-principles calculations. Moreover, we have resolved the trigonal warping of the valence band near the $\bar{K}$ point of the BZ in the bulk materials. This effect has been theoretically predicted in monolayer TMDCs and has been attributed to an anisotropic correction of order $q^{3}$ to the energy. This warping effect reflects the threefold rotational symmetry of the underlying crystal lattice ${ }^{39,40}$. From symmetry considerations, the same is expected to happen for bulk TMDCs as well, as we have shown in our measurements.
Furthermore, we have provided the first momentum-resolved experimental evidence of the spin-split valence band of monolayer $\mathrm{MoS}_{2}$ grown on HOPG substrate. However, further spinresolved ARPES measurements are needed to confirm the spin configuration of these two split bands. According to our calculations, the maximal splitting occurs at $\bar{K}$, and is about $148 \mathrm{meV}$. The magnitude of the splitting depends on the momentum. The splitting curve rises sharply near $k=0.6 \AA^{-1}$, and approaches to its maximum with a smaller slope at larger $k$. This momentum dependence of spin splitting can be attributed to the changes in the orbital components of the states in the valence and conduction bands (see Supplementary Note 1 and Supplementary Figs 5 and 6 for details).

Recently, experimental evidence and theoretical prediction of valley confinement has been suggested in monolayer $\mathrm{MoS}_{2}$. Specifically, it has been suggested that the split valence band at neighbouring $\bar{K}$ points of the $\mathrm{BZ}\left(\bar{K}\right.$ and $\left.\bar{K}^{\prime}\right)$ posses opposite out-of-plane spin configurations ${ }^{22-24,32}$. The spin-orbit splitting observed in our study of monolayers of $\mathrm{MoS}_{2}$ paves the way to more detailed studies of spin and valley physics in TMDCs. 
a

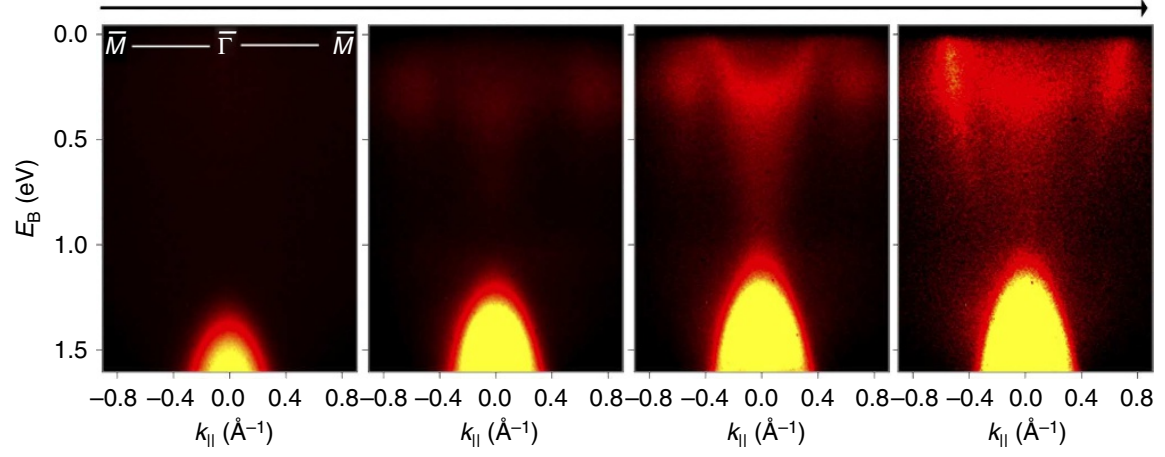

b

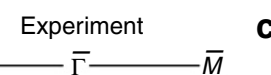

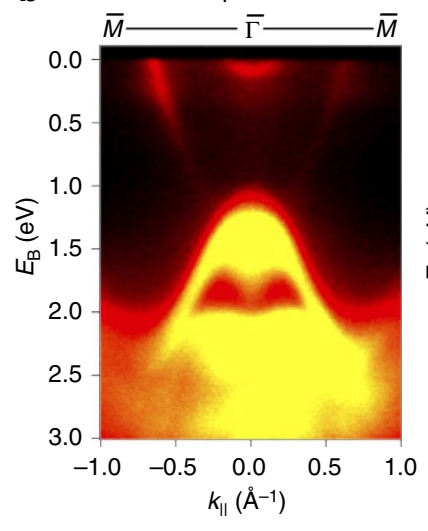

d

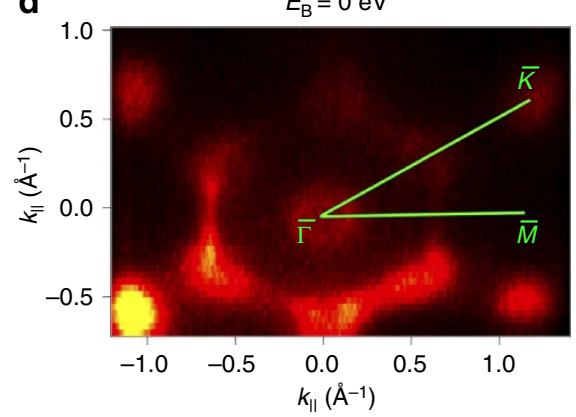

C Calculation

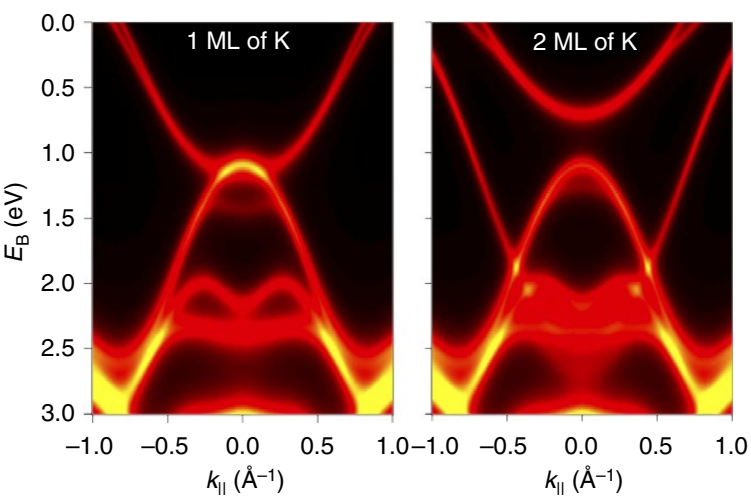

e

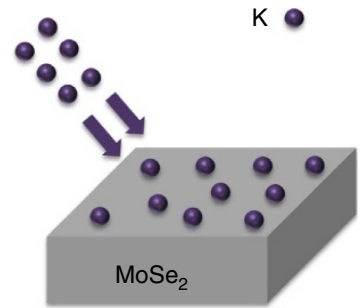

Figure 4 | Quantum well states (QWSs) on TMDCs through surface potassium deposition. (a) Evolution of the low-energy electronic structure of MoSe $e_{2}$ during in situ surface potassium deposition as measured by ARPES, showing the process of formation of in-gap quantum well states. (b) ARPES band structure spectra upon the completion of the potassium deposition process. (c) First-principles calculated band structure of one and two atomic layers of potassium on the surface of bulk $\mathrm{MoSe}_{2}$, indicating the formation of two QWSs in the ARPES measurements. (d) The Fermi surface obtained after the potassium deposition process is completed, featuring the observation of two QWSs in the form of two electron-like circular pockets around $\bar{\Gamma}$, as well as the surface states of potassium at $\bar{K}$ points. The green lines represent the $\bar{\Gamma}-\bar{M}$ and $\bar{\Gamma}-\bar{K}$ directions of the BZ. Here the ARPES measurements were performed at photon energy of $h v=70 \mathrm{eV}$ and temperature of $T=60 \mathrm{~K}$. (e) Illustration of the in situ surface potassium deposition.

It also serves as a guide for spintronics and valleytronics applications of TMDCs.

Our results also track the evolution of the band structure of $\mathrm{MoSe}_{2}$ throughout the surface deposition process with an alkali metal (potassium). We note that short after the deposition process has started potassium atoms sit on the surface of $\mathrm{MoSe}_{2}$ in a manner that results to the partial formation of the first QWS (see second panel in Fig. 4a). Consequently, the first QWS appears in full, as shown in the third panel of Fig. 4a, and finally the second quantum well band is formed partially close to the Fermi level. The QWSs in the epitaxial potassium layers can be considered as being confined by vacuum barrier on one side and the bulk band gap of $\mathrm{MoSe}_{2}$ on the other side ${ }^{45}$. When the energy of the QWS is within the bulk band gap of $\mathrm{MoSe}_{2}$, the state is well trapped in the epitaxial layer (for more details see Supplementary Note 1). In the process of surface deposition, 2D conduction channels (QWSs) emerge on the surface of the semiconducting $\mathrm{MoSe}_{2}$ (with an indirect insulating energy gap of $\sim 1.8 \mathrm{eV}$ ) and, as a consequence, the electronic behaviour of the system changes dramatically. This tunable feature can be utilized to quantitatively control the electronic properties of this quasi-2D material. Our investigation of the dynamical process in which a nearly free 2DEG is formed on TMDCs could have important implications for future optoelectronics studies and applications of TMDCs using surface manipulations by elements such as alkali metals on bulk, few-layers and monolayers of these materials.

In conclusion, we have conducted detailed high-resolution ARPES and first-principles calculations studies of the electronic 
structure of both bulk and monolayer TMDCs. We have resolved the two distinct spin-degenerate valence bands of the bulk $\mathrm{MoSe}_{2}$, and have provided evidence for the spin-orbit split bands of the monolayer $\mathrm{MoS}_{2}$, at the corners of the BZ. We have furthermore investigated the process of the formation of QWSs on the surface of bulk $\mathrm{MoSe}_{2}$, and demonstrated the formation of a 2DEG on the cleaved surfaces of TMDCs. These results will be extremely valuable in designing, fabricating and optimizing these materials for future device applications.

Finally, we note that after the submission of this manuscript, another group reported the splitting in the valence band of $\mathrm{MoSe}_{2}$ monolayer and few-layers films, but neither trigonal warping effect nor QWSs on the bulk $\mathrm{MoSe}_{2}$ were reported there ${ }^{46}$.

\section{Methods}

Electronic structure measurements. ARPES measurements were performed with incident photon energies of $35-100 \mathrm{eV}$ at beamline 4.0.3 of the Advanced Light Source in the Lawrence Berkeley National Laboratory. Samples were cleaved in situ between 10 and $20 \mathrm{~K}$ at chamber pressure better than $5 \times 10^{-11}$ Torr resulting in shiny surfaces. Energy resolution was better than $15 \mathrm{meV}$ and momentum resolution was better than $1 \%$ of the surface $\mathrm{BZ}$.

Sample growth and characterization. To prepare $\mathrm{MoS}_{2}$ films, molybdenum chloride $\left(\mathrm{MoCl}_{5}\right)$ and sulphur powder were used as precursors and the growth of monolayer films was carried out in a CVD chamber. The ultrasonicated cleaned HOPG substrates were loaded into the CVD chamber $6 \mathrm{~cm}$ away from the central hot zone. Argon (Ar) gas was used as carrier gas in a pressure of 3 Torr (ref. 47). Single crystals of $\mathrm{MoSe}_{2}$ were grown by the chemical vapour transport method, using $\mathrm{I}_{2}$ as the transporting agent. They were formed in silver-coloured, graphitelike, single-crystalline platelets up to $10 \times 10 \mathrm{~mm}^{2}$ in surface area and $2 \mathrm{~mm}$ in thickness (inset in Fig. 1a). X-ray photoelectron spectroscopy measurements were performed by means of Physical Electronics 5500 photoelectron spectrometer. All spectra were collected using Mg Ka X-ray operating at $300 \mathrm{~W}$. The binding energy scale was calibrated by setting the main component of the $\mathrm{C} 1 \mathrm{~s}$ peak at $284.6 \mathrm{eV}$.

First-principles calculation methods. First-principles calculations of the electronic structures were performed using HGH-type pseudo-potentials ${ }^{48}$ and a plane-wave basis set. The main programme employed was developed by the ABINIT group ${ }^{49,50}$. Spin-orbit coupling was included, where appropriate, using the relativistic local-density approximation (LDA). The experimental resolution was taken into account in the spectral simulations of Figs 2-4, but the matrix element effect was not considered (see Supplementary Note 2 and Supplementary Figs 7 and 8 for more details).

\section{References}

1. Geim, A. K. \& Novoselov, K. S. The rise of graphene. Nat. Mater. 6, 183-191 (2007).

2. Geim, A. K. \& Kim, P. Carbon wonderland. Sci. Am. 298, 90-97 (2008).

3. Hasan, M. Z. \& Kane, C. L. Colloquium: topological insulators. Rev. Mod. Phys. 82, 3045-3067 (2010).

4. Qi, X.-L. \& Zhang, S.-C. Topological insulators and superconductors. Rev. Mod. Phys. 83, 1057-1110 (2011)

5. Hsieh, D. et al. A topological Dirac insulator in a quantum spin Hall phase. Nature 452, 970-974 (2008).

6. Xia, Y. et al. Observation of a large-gap topological-insulator class with a single Dirac cone on the surface. Nat. Phys. 5, 398-402 (2009).

7. $\mathrm{Xu}, \mathrm{S}$.-Y. et al. Topological phase transition and texture inversion in a tunable topological insulator. Science 332, 560-564 (2011).

8. Xu, S.-Y. et al. Observation of a topological crystalline insulator phase and topological phase transition in $\mathrm{Pb}_{1-x} \mathrm{Sn}_{x}$ Te. Nat. Commun. 3, 1192 (2012).

9. Wang, Q. H., Kalantar-Zadeh, K., Kis, A., Coleman, J. N. \& Strano, M. S. Electronics and optoelectronics of two-dimensional transition metal dichalcogenides. Nat. Nanotechnol. 7, 699-712 (2012).

10. Balendhran, S. et al. Two-dimensional molybdenum trioxide and dichalcogenides. Adv. Funct. Mater. 23, 3952-3970 (2013).

11. Coleman, J. N. et al. Two-dimensional nanosheets produced by liquid exfoliation of layered materials. Science 331, 568-571 (2011).

12. Castellanos-Gomez, A. et al. Laser-thinning of $\mathrm{MoS}_{2}$ : on demand generation of a single-layer semiconductor. Nano Lett. 12, 3187-3192 (2012).

13. Kobayashi, K. \& Yamauchi, J. Electronic structure and scanning-tunnelingmicroscopy image of molybdenum dichalcogenide surfaces. Phys. Rev. B 51, 17085-17095 (1995).

14. Lebègue, S. \& Eriksson, O. Electronic structure of two-dimensional crystals from $a b$ initio theory. Phys. Rev. B 79, 115409 (2009).
15. Ding, Y. et al. First principles study of structural, vibrational and electronic properties of graphene-like $\mathrm{MM}_{2}(\mathrm{M}=\mathrm{Mo}, \mathrm{Nb}, \mathrm{W}, \mathrm{Ta} ; \mathrm{X}=\mathrm{S}, \mathrm{Se}, \mathrm{Te})$ monolayers. Physica B Cond. Matt. 406, 2254-2260 (2011).

16. Coehoorn, R. et al. Electronic structure of MoSe2, MoS2 and WSe2. I. Band structure calculations and photoelectron spectroscopy. Phys. Rev. B 35, 6195-6202 (1987).

17. Kuc, A., Zibouche, N. \& Heine, T. Influence of quantum confinement on the electronic structure of the transition metal sulfide $T_{2}$. Phys. Rev. B 83, 245213 (2011).

18. Mak, K. F., Lee, C., Hone, J., Shan, J. \& Heinz, T. F. Atomically thin $\mathrm{MoS}_{2}$ : a new direct-gap semiconductor. Phys. Rev. Lett. 105, 136805 (2010).

19. Splendiani, A. et al. Emerging photoluminescence in monolayer $\mathrm{MoS}_{2}$. Nano Lett. 10, 1271-1275 (2010).

20. Podzorov, V., Gershenson, M. E., Kloc, C., Zeis, R. \& Bucher, E. High-mobility field-effect transistors based on transition metal dichalcogenides. Appl. Phys. Lett. 84, 3301-3303 (2004)

21. Zhu, Z. Y., Cheng, Y. C. \& Schwingenschlögl, U. Giant spin-orbit-induced spin splitting in two-dimensional transition-metal dichalcogenide semiconductors. Phys. Rev. B 84, 153402 (2011).

22. Xiao, D., Liu, G.-B., Feng, W., Xu, X. \& Yao, W. Coupled spin and valley physics in monolayers of $\mathrm{MoS}_{2}$ and other group-VI dichalcogenides. Phys. Rev. Lett. 108, 196802 (2012).

23. Zeng, H., Dai, J., Yao, W., Xiao, D. \& Cui, X. Valley polarization in $\mathrm{MoS}_{2}$ monolayers by optical pumping. Nat. Nanotechnol. 7, 490-493 (2012).

24. Zeng, H. et al. Optical signature of symmetry variations and spin-valley coupling in atomically thin tungsten dichalcogenides. Sci. Rep. 3, 1608 (2013)

25. Radisavljevic, B., Radenovic, A., Brivio, J., Giacometti, V. \& Kis, A. Single-layer $\mathrm{MoS}_{2}$ transistors. Nat. Nanotechnol. 6, 147-150 (2011).

26. Yoon, Y., Ganapathi, K. \& Salahuddin, S. How good can monolayer $\mathrm{MoS}_{2}$ transistors be? Nano Lett. 11, 3768-3773 (2011).

27. Yin, Z. et al. Single-layer $\mathrm{MoS}_{2}$ phototransistors. ACS Nano 6, 74-80 (2012)

28. Zhang, Y., Ye, J., Matsuhashi, Y. \& Iwasa, Y. Ambipolar $\mathrm{MoS}_{2}$ thin flake transistors. Nano Lett. 12, 1136-1140 (2012).

29. Frindt, R. F. The optical properties of single crystals of $\mathrm{WSe}_{2}$ and $\mathrm{MoTe}_{2}$ J. Phys. Chem. Solids 24, 1107-1108 (1963).

30. Frindt, R. F. \& Yoffe, A. D. Physical properties of layer structures: optical properties and photoconductivity of thin crystals of molybdenum disulphide. Proc. R. Soc. Lond Ser. A 273, 69-83 (1963).

31. Kam, K. K. \& Parkinson, B. A. Detailed photocurrent spectroscopy of the semiconducting group VIB transition metal dichalcogenides. J. Phys. Chem. A 86, 463-467 (1982)

32. Mak, K. F., He, K., Shan, J. \& Heinz, T. F. Control of valley polarization in monolayer $\mathrm{MoS}_{2}$ by optical helicity. Nat. Nanotechnol. 7, 494-498 (2012)

33. Böker, Th. et al. Band structure of $\mathrm{MoS}_{2}, \mathrm{MoSe}_{2}$ and $\alpha-\mathrm{MoTe}_{2}$ : angle-resolved photoelectron spectroscopy and $a b$ initio calculations. Phys. Rev. B 64, 235305 (2001).

34. Mahatha, S. K., Patel, K. D. \& Menon, K. S. R. Electronic structure investigation of $\mathrm{MoS}_{2}$ and $\mathrm{MoSe}_{2}$ using angle-resolved photoemission spectroscopy and $a b$ initio band structure studies. J. Phys. Condens. Matter 24, 475504 (2012).

35. Mahatha, S. K. \& Menon, K. S. R. Inhomogeneous band bending on $\mathrm{MoS}_{2}(0001)$ arising from surface steps and dislocations. J. Phys. Condens. Matter 24, 305502 (2012).

36. Mahatha, S. K. \& Menon, K. S. R. Quantum well states in Ag thin films on $\mathrm{MoS}_{2}$ (0001) surfaces. J. Phys.: Condens. Matter 25, 115501 (2013).

37. Jin, W. et al. Direct measurement of the thickness-dependent electronic band structure of $\mathrm{MoS}_{2}$ using angle-resolved photoemission spectroscopy. Phys. Rev. Lett. 111, 106801 (2013).

38. Zahid, F., Liu, L., Zhu, Y., Wang, J. \& Guo, H. A generic tight-binding model for monolayer, bilayer and bulk $\mathrm{MoS}_{2}$. AIP Adv. 3, 052111 (2013).

39. Kormányos, A. et al. Monolayer $\mathrm{MoS}_{2}$ : trigonal warping, the $\Gamma$ valley, and spin orbit coupling effects. Phys. Rev. B 88, 045416 (2013).

40. Rostami, H., Moghaddam, A. G. \& Asgari, R. Effective lattice Hamiltonian for monolayer $\mathrm{MoS}_{2}$ : tailoring electronic structure with perpendicular electric and magnetic fields. Phys. Rev. B 88, 085440 (2013).

41. Chang, C.-H., Fan, X., Lin, S.-H. \& Kuo, J.-L. Orbital analysis of electronic structure and phonon dispersion in $\mathrm{MoS}_{2}, \mathrm{MoSe}_{2}, \mathrm{WS}_{2}$, and $\mathrm{WSe} \mathrm{S}_{2}$ monolayers under strain. Phys. Rev. B 88, 195420 (2013).

42. Zhang, P. et al. A precise method for visualizing dispersive features in image plots. Rev. Sci. Instrum 82, 043712-043712 (2011).

43. Hüfner, S. Photoelectron Spectroscopy: Principles and Applications (Springer, Heidelberg, 2003).

44. Dolui, K., Rungger, I., Das Pemmaraju, C. \& Sanvito, S. Possible doping strategies for $\mathrm{MoS}_{2}$ monolayers: an ab initio study. Phys. Rev. B 88, 075420 (2013).

45. Chiang, T.-C. Photoemission studies of quantum well states in thin films. Surf. Sci. Rep. 39, 181-235 (2000).

46. Zhang, Y. et al. Direct observation of the transition from indirect to direct bandgap in atomically thin epitaxial $\mathrm{MoSe}_{2}$. Nat. Nanotechnol. 9, 111-115 (2014) 
47. Yu, Y. et al. Controlled scalable synthesis of uniform, high-quality monolayer and few-layer $\mathrm{MoS}_{2}$ films. Sci. Rep. 3, 1866 (2013).

48. Hartwigsen, C., Goedecker, S. \& Hutter, J. Relativistic separable dual-space Gaussian pseudopotentials from H to Rn. Phys. Rev. B 58, 3641-3662 (1998).

49. Gonze, X. et al. First-principles computation of material properties: the ABINIT software project. Comput. Mater. Sci. 25, 478-492 (2002)

50. Gonze, X., Rignanese, G.-M. \& Caracas, R. First-principle studies of the lattice dynamics of crystals, and related properties. Z. Kristallogr. 220, 458-472 (2005).

\section{Acknowledgements}

Work at Princeton University is supported by the US National Science Foundation Grant, NSF-DMR-1006492. M.Z.H. acknowledges visiting-scientist support from Lawrence Berkeley National Laboratory and additional partial support from the A.P. Sloan Foundation and NSF-DMR-0819860. The photoemission measurements using synchrotron X-ray facilities are supported by the Basic Energy Sciences of the US Department of Energy. Theoretical computations are supported by the US Department of Energy (supported by DE-FG02-05ER46200 and Feynman cluster at Princeton University, Department of Physics). Sample growth and characterization are partially supported by DMR-0819860, DMR-1006492 and by the National Science Council (NSC), Taiwan, under project number NSC100-2119-M- 002-021.

\section{Author contributions}

N.A. and M.Z.H. conceived and designed the experiments. N.A. performed the experiments with assistance from S.-Y.X., M.N., C.L., I.B. and J.D.D.; G.B. performed DFT calculations and spectral simulations; N.A., G.B. and M.Z.H. performed data analysis, figure planning and draft preparation; D.-X.Q., R.S. and F.-C.C. provided samples and performed sample characterization; M.Z.H. was responsible for the overall direction, planning and integration among different research units.

\section{Additional information}

Supplementary Information accompanies this paper at http://www.nature.com/ naturecommunications

Competing financial interests: The authors declare no competing financial interests

Reprints and permission information is available online at http://npg.nature.com/ reprintsandpermissions/

How to cite this article: Alidoust, N. et al. Observation of monolayer valence band spin-orbit effect and induced quantum well states in $\mathrm{MoX}_{2}$. Nat. Commun. 5:4673 doi: $10.1038 /$ ncomms5673 (2014). 


\section{Erratum: Observation of monolayer valence band spin-orbit effect and induced quantum well states in $\mathrm{MoX}_{2}$}

Nasser Alidoust, Guang Bian, Su-Yang Xu, Raman Sankar, Madhab Neupane, Chang Liu, Ilya Belopolski, Dong-Xia Qu, Jonathan D. Denlinger, Fang-Cheng Chou \& M. Zahid Hasan

Nature Communications 5:4673 doi: 10.1038/ncomms5673 (2014); Published 22 Aug 2014; Updated 13 Oct 2014

The original version of this Article contained a typographical error in the first sentence of the abstract, where the phrase 'transition metal dichalcogenides' was inadvertently duplicated. This has now been corrected in both the PDF and HTML versions of the Article. 